\title{
Measurement of refractive index of hemoglobin in the visible/NIR spectral range
}

Ekaterina N. Lazareva

Valery V. Tuchin 


\title{
Measurement of refractive index of hemoglobin in the visible/NIR spectral range
}

\author{
Ekaterina N. Lazareva ${ }^{a, b, *}$ and Valery V. Tuchin ${ }^{a, c, d}$ \\ a Saratov State University (National Research University), Research Educational Institute of Optics and Biophotonics, Saratov, Russia \\ 'Immanuel Kant Baltic Federal University, Center for Functionalized Magnetic Materials (FunMagMa), Kaliningrad, Russia \\ ${ }^{c}$ Tomsk State University (National Research University), Interdisciplinary Laboratory of Biophotonics, Tomsk, Russia \\ Institute of Precision Mechanics and Control RAS, Laboratory of Laser Diagnostics of Technical and Living Systems, Saratov, Russia
}

\begin{abstract}
This study is focused on the measurements of the refractive index of hemoglobin solutions in the visible/near-infrared (NIR) spectral range at room temperature for characteristic laser wavelengths: 480, $486,546,589,644,656,680,930,1100,1300$, and $1550 \mathrm{~nm}$. Measurements were performed using the multiwavelength Abbe refractometer. Aqua hemoglobin solutions of different concentrations obtained from human whole blood were investigated. The specific increment of refractive index on hemoglobin concentration and the Sellmeier coefficients were calculated. ๑ 2018 Society of Photo-Optical Instrumentation Engineers (SPIE) [DOI: 10.1117/1.JBO .23.3.035004]
\end{abstract}

Keywords: hemoglobin; refractive index; dispersion; Sellmeier coefficients; specific refraction increment.

Paper 170750R received Nov. 21, 2017; accepted for publication Feb. 20, 2018; published online Mar. 15, 2018.

\section{Introduction}

The refractive index (RI) of biological tissue is a basic material parameter that characterizes how light interacts with tissue. ${ }^{1}$ In many optical studies, a rough estimate of RI of the tissue under study, based on the fact that the main constituent of tissue is salt water-filled cells or more precisely a mixture of salt water and proteins, is often used. ${ }^{2,3}$ For many tissues and blood components, the data for RI in a wide spectral range and concentrations are not yet available. ${ }^{4}$

The study of optical properties of hemoglobin is important for the development of diagnostic and laser treatment techniques, where consideration of blood optical properties is critical. Various optical methods widely used for tissue characterization, such as visible and near-infrared (NIR) spectroscopy, optical coherence tomography, and fluorescence spectroscopy, need exact data for RI of tissue, blood, and their components to quantify properly experimental data. ${ }^{5-11}$

At present, the usage of RI as a diagnostic marker is urgent. ${ }^{5-20}$ Sometimes, it is a self-sufficient parameter for tissue and blood characterization. Zhernovaya et al. considered the change of RI of hemoglobin and albumin at the interaction with glucose as a possible method for studying the glycation process and determining glycated proteins, which is important for the monitoring of diabetes mellitus. ${ }^{9}$ The RI of tissue was used as a marker for cancer, reflecting changes of optical properties in the course of pathology development. ${ }^{10,11,14-20}$ An additional motivation for this study is a lot of discrepancies between the RIs reported in the literature by different research groups.

The RI is a complex value consisting of a real part $n$, which represents the ratio of the speed of light in a vacuum to the speed of light in the material $n=c / v$, and an imaginary part $k$, which represents light attenuation ${ }^{21-25}$

*Address all correspondence to: Ekaterina N. Lazareva, E-mail: Lazarevaen@ list.ru $\tilde{n}=n+i k$

Because of tissue heterogeneity, $n$ is always known as the effective or average RI. ${ }^{24,26}$ According to the classical theory of light dispersion, the components of the complex RI of molecular structures can be written as ${ }^{21-25}$

$$
\begin{aligned}
& n=1+\frac{2 \pi q^{2} N\left(\omega_{0}^{2}-\omega^{2}\right)}{m\left(\omega_{0}^{2}-\omega^{2}\right)^{2}+\gamma^{2} \omega^{2}}, \\
& k=\frac{2 \pi q^{2} N \gamma \omega}{m\left(\omega_{0}^{2}-\omega^{2}\right)^{2}+\gamma^{2} \omega^{2}},
\end{aligned}
$$

where $q$ is the molecular charge, $N$ is the number of molecules per unit volume, $m$ is the molecular mass, $\omega$ is the probing light frequency, $\omega_{0}$ is the central frequency of molecular absorption band, and $\gamma$ is the attenuation coefficient. ${ }^{21-25}$

Over the last decades, various techniques to determine RI of biological tissues were developed; they include confocal microscopy, ${ }^{1,6}$ optical fiber cladding method, ${ }^{27}$ minimum deviation angle method, ${ }^{28,29}$ optical coherent tomography with multiple modifications, ${ }^{6,9,30-36}$ total internal reflection method, ${ }^{26,37,38}$ measurement of the intensity profile of diffuse light refracted into the prism around the critical angle, ${ }^{39}$ various modifications of nonlinear phase microscopy, ${ }^{40-42}$ and quantitative phase imaging techniques. ${ }^{12,43,44}$

Because of the strong hemoglobin absorption, direct measurements of the real part of RI using conventional refractometers (for example, an Abbe refractometer) have proven to be difficult, and data are available at a few wavelengths only. In an early study, for example, Barer measured $n$ for solutions of oxygenated hemoglobin at $589 \mathrm{~nm}$ only. ${ }^{44} \mathrm{He}$ also discussed RI dependence on the hemoglobin concentration and presented the expression

$1083-3668 / 2018 / \$ 25.00$ ๑ 2018 SPIE 
$n=n_{\mathrm{H}_{2} \mathrm{O}}+\alpha C$

where $n_{\mathrm{H}_{2} \mathrm{O}}$ is the RI of distilled water, $C$ is the concentration of hemoglobin, and $\alpha$ is the specific refraction increment. ${ }^{44}$

Faber et al. measured the RI of solutions of oxygenated and deoxygenated hemoglobin at $800 \mathrm{~nm} .^{45}$ Friebel and Meinke measured directly the RI of solutions of oxygenated hemoglobin at $633 \mathrm{~nm}$ for several concentrations $\mathrm{s}^{7,8}$

$n=n_{\mathrm{H}_{2} \mathrm{O}}(1+\beta C)$.

Zhernovaya et al. also used the formula similar to Eq. (4) to describe the linear dependence of the RI of hemoglobin on the concentration

$n=n_{0}+\alpha C$,

where $n_{0}$ is the RI of solvent, $C$ is the hemoglobin concentration in $\mathrm{dl} / \mathrm{g}$, and $\alpha$ is the specific refraction increment. ${ }^{4,46}$

Jin et al. measured RI of hemoglobin solution at 633 and $532 \mathrm{~nm}$ using a total internal reflection technique. ${ }^{37}$ Park et al. measured the dispersion of $\mathrm{Hb}$ solutions, prepared from $\mathrm{Hb}$ protein powder, at $440,546,560,580,600,655$, and $700 \mathrm{~nm}$ using spectroscopic phase microscopy. ${ }^{41}$ Deng et al. showed that, in the 400 to $750 \mathrm{~nm}$ range, hemoglobin solution is characterized by specific forms of dispersion and extinction spectra. ${ }^{47}$ Yahya and Saghir measured RIs for multiple temperatures and wavelengths using the Abbemat refractometer. ${ }^{48}$ They found linear dependences of RI on hemoglobin concentration and temperature and nonlinear on the wavelength.

Analysis of the dispersion relation in similar studies showed significant differences for oxyhemoglobin and deoxyhemoglobin, related to the difference in the imaginary part of the RI for the 500 to $600 \mathrm{~nm}$ region. ${ }^{4,45-50}$ There is lack of data for RI of hemoglobin solutions for concentrations close to that in the red blood cells (RBC), especially for the NIR region.

This study is focused on the determination of the RI of hemoglobin in the visible and NIR ranges at room temperature, aiming for further quantification dispersion of hemoglobin solutions. Measurements were carried out using the multiwavelength Abbe refractometer (Atago, Japan). The hemoglobin solutions of different concentrations obtained from human whole blood were investigated. The RI of hemoglobin solutions was measured for the wavelengths: 480, 486, 546, 589, 644, $656,680,930,1100,1300$, and $1550 \mathrm{~nm}$, which are characteristic for different lasers widely used in biomedicine. The specific increment of RI and Sellmeier coefficients for dispersion on hemoglobin concentration were calculated based on the experimental data.

\section{Methods and Materials}

Hemoglobin obtained from human whole blood was used to prepare hemoglobin specimens. Whole blood was drawn from the human vein. Immediately after collecting blood into a test tube, heparin was added in it. The sample of blood from a healthy person was taken at the State Healthcare Organization "Saratov City Clinical Hospital No. 2 named after V. I. Razumovsky" with the permission of the volunteer. To separate blood into fractions, the centrifugation for $10 \mathrm{~min}$ at $2000 \mathrm{rpm}$ and at room temperature was provided. This resulted in separation of blood plasma, leuko-platelet layer, and RBC suspension. To conduct hemolysis and preparation of hemoglobin solutions, RBC suspension was separated and

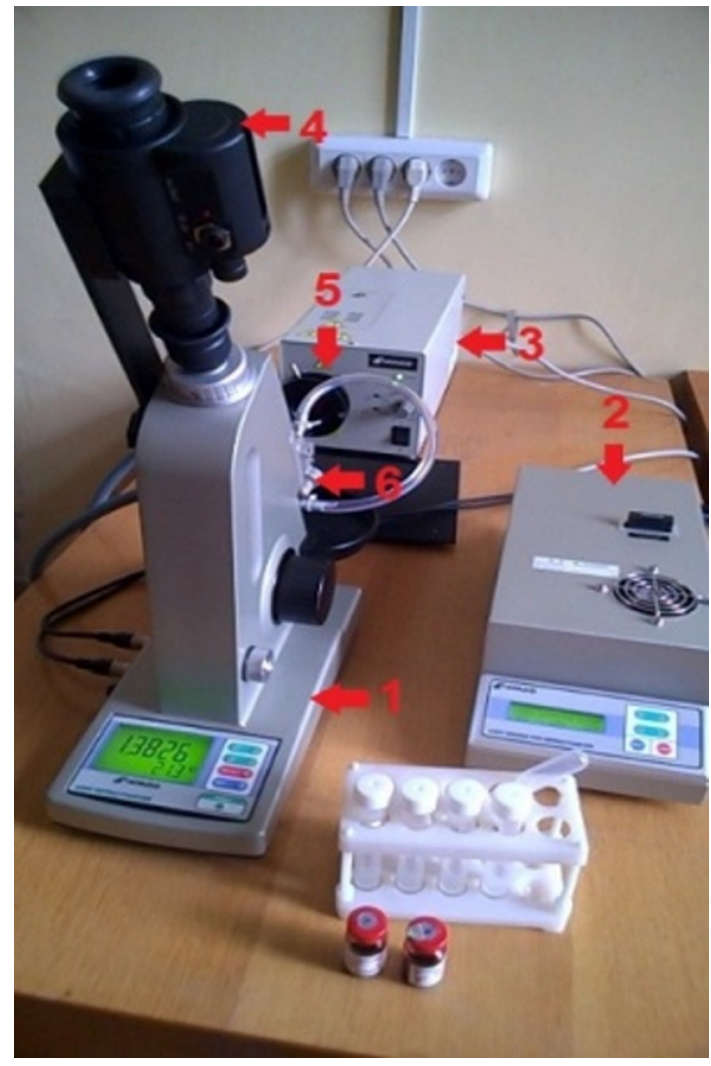

Fig. 1 General view of the multiwavelength Abbe refractometer (Atago, Japan): 1, refractometer; 2,--power supply; 3, light source; 4 , the eyepiece imager for measurements in the NIR region; 5 , interference filter; and 6, sample.

placed in a vial for freezing in a freezer at a temperature of $-15^{\circ} \mathrm{C}$ for $24 \mathrm{~h}$.

Actual concentration of the basic hemoglobin solution was estimated by the spectral technique and amounted to $260 \mathrm{~g} / 1$. In the experiment, we measured the RI of three specimens taken from the same sample. The RI measurements for solutions of different concentrations obtained by diluting the basic solution of hemoglobin in saline solution were also provided.

At measurements, a sample layer on the working surface of the prism had a small thickness of about 20 to $30 \mu \mathrm{m}$. The time of the full oxygenation (78.4\% to $94.2 \%$ ) of hemoglobin in such a layer is about 6 to $10 \mathrm{~s} .{ }^{51}$ Therefore, hemoglobin is fully saturated with oxygen, and the process of oxygenation during measurements is expected to not affect the result.

Measurements were performed using the multiwavelength Abbe refractometer (Atago, Japan) (Fig. 1). The RI was measured for samples of hemoglobin obtained from human whole blood $(65,87,173$, and $260 \mathrm{~g} / \mathrm{l})$ on 11 wavelengths from 480 to $1550 \mathrm{~nm}$. The temperature was $23^{\circ} \mathrm{C}$.

Multiwavelength refractometer Abbe allows one to measure the RI in the wavelength range of 450 to $1550 \mathrm{~nm}$ with an accuracy of \pm 0.0002 . The working principle of the refractometer technique is based on determining the critical angle of the total reflection, where the incident light waves are completely reflected with a 90-deg angle to the normal position. The incident light waves with angles greater than the critical angle will only experience reflection at the interface surface and no refraction will be observed. The total internal reflection method is applicable to measurement of the RI of biological media, 


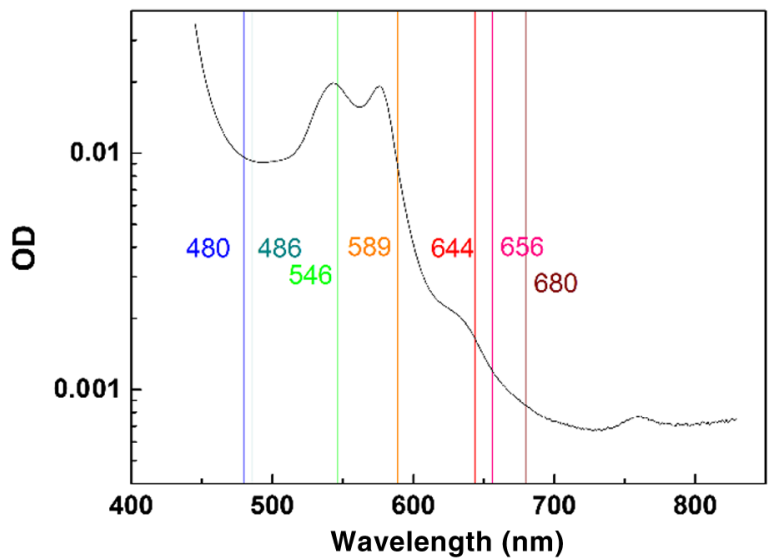

Fig. 2 The optical density spectrum of a solution of hemoglobin $260 \mathrm{~g} / \mathrm{l}$. By the vertical lines visible working wavelengths of Atago refractometer are shown.

which are characterized by high light scattering and absorption. The wavelength of the light source is determined by the selection of the particular interferential filter. Available interferential filters allowed for measurements on the wavelengths $480 \pm 2$, $486 \pm 2,546 \pm 2,589 \pm 2,644 \pm 2$, and $656 \pm 2 \mathrm{~nm}, 680 \pm 5$, $930 \pm 6, \quad 1100 \pm 26, \quad 1300 \pm 25$, and $1550 \pm 25 \mathrm{~nm}$. The calibration of the device by measuring RI of distilled water at a wavelength of $589 \mathrm{~nm}$ (the absorption band of sodium) was used at the beginning of each experiment. The average measurement error of the RI was \pm 0.0003 .

To approximate the dispersion dependence of the RI of the hemoglobin solution, the Sellmeier formula was used

$n^{2}(\lambda)=1+\frac{A 1 * \lambda^{2}}{\lambda^{2}-B 1}+\frac{A 2 * \lambda^{2}}{\lambda^{2}-B 2}$,

where $A 1, A 2, B 1$, and $B 2$ are empirical constants. Sellmeier's formula gives a good agreement for describing the dispersion dependence of the RI of multicomponent systems near absorption bands of a medium under study. ${ }^{52}$ Mathematical calculations were performed in the software package Origin ProLab.

\section{Measurement Results}

The optical density spectra of a solution of hemoglobin obtained from the whole blood by hemolysis are shown in Fig. 2. The graph shows that the wavelengths available for RI measurements, i.e., 480, 486, 546, 589, 644, and $656 \mathrm{~nm}$, belong to different or the same absorption bands of hemoglobin with quite different absorption abilities. Therefore, we can expect different inclusion of anomalous dispersion in RI wavelength dependence at these wavelengths. Wavelength $546 \mathrm{~nm}$ is the closest to the isobestic point $544 \mathrm{~nm}$, where the absorption of hemoglobin does not depend on the degree of oxygenation. ${ }^{53}$

Table 1 presents data for Atago refractometer measurements of RI for four different concentrations of hemoglobin, i.e., 65, 87,173 , and $260 \mathrm{~g} / 1$ at room temperature, $23^{\circ} \mathrm{C}$.

It is well known that the RI of proteins is nonlinearly dependent on the wavelength. ${ }^{4,45-50,54}$ Figure 3 shows the dispersion curves for hemoglobin solutions in the visible/NIR spectral range. The symbols are experimental data from Table 1, and the lines correspond to the fit of these data to the Sellmeier formula, Eq. (7). Table 2 presents data for the decomposition of the Sellmeier formula.

As it follows from Table 2, for all wavelengths and hemoglobin concentrations, measured RIs are well fit to the Sellmeier formula with correlation coefficient, $R^{2}$, equal or better than 0.993. Specifically, there is a linear relationship between the RI and hemoglobin concentration. The RI of the hemoglobin samples is also temperature-dependent, although the temperature effect on the RI is small when compared with the hemoglobin concentration effect. Figure 4 shows the dependence of the $\mathrm{RI}$ of human hemoglobin solution on hemoglobin concentration for the room temperature of $23^{\circ} \mathrm{C}$. These data can be used to predict the hemoglobin concentration of the blood sample based on the knowledge of the RI and using the refraction increment provided. This dependence can be described by Eqs. (4) and (5).

Table $1 \mathrm{RI}$ measured for four different concentrations of hemoglobin at room temperature $23^{\circ} \mathrm{C}$. SD is shown in brackets.

\begin{tabular}{lccccc}
$\lambda(\mathrm{nm})$ & $0 \mathrm{~g} / \mathrm{l}$ & $65 \mathrm{~g} / \mathrm{l}$ & $87 \mathrm{~g} / \mathrm{l}$ & $173 \mathrm{~g} / \mathrm{l}$ & $260 \mathrm{~g} / \mathrm{l}$ \\
\hline 480 & $1.3371(0.0003)$ & $1.3476(0.0003)$ & $1.3571(0.0003)$ & $1.3728(0.0003)$ & $1.3879(0.0002)$ \\
486 & $1.3371(0.0002)$ & $1.3478(0.0002)$ & $1.3563(0.0002)$ & $1.3721(0.0002)$ & $1.3871(0.0004)$ \\
546 & $1.3342(0.0002)$ & $1.3448(0.0002)$ & $1.3533(0.0002)$ & $1.3681(0.0007)$ & $1.3836(0.0002)$ \\
589 & $1.3329(0.0002)$ & $1.3438(0.0002)$ & $1.3519(0.0003)$ & $1.3667(0.0004)$ & $1.3821(0.0004)$ \\
644 & $1.3313(0.0002)$ & $1.3419(0.0002)$ & $1.3497(0.0002)$ & $1.3640(0.0003)$ & $1.3801(0.0003)$ \\
656 & $1.3308(0.0002)$ & $1.3414(0.0002)$ & $1.3493(0.0002)$ & $1.3647(0.0003)$ & $1.3792(0.0009)$ \\
680 & $1.3301(0.0002)$ & $1.3403(0.0003)$ & $1.3482(0.0003)$ & $1.3633(0.0003)$ & $1.3771(0.0002)$ \\
930 & $1.3259(0.0002)$ & $1.3360(0.0002)$ & $1.3440(0.0002)$ & $1.3572(0.0003)$ & $1.3735(0.0007)$ \\
1100 & $1.3222(0.0002)$ & $1.3329(0.0002)$ & $1.3411(0.0002)$ & $1.3542(0.0002)$ & $1.3690(0.0006)$ \\
1300 & $1.3174(0.0002)$ & $1.3280(0.0005)$ & $1.3364(0.0002)$ & $1.3503(0.0002)$ & $1.3642(0.0004)$ \\
1550 & $1.3140(0.0002)$ & $1.3244(0.0004)$ & $1.3314(0.0003)$ & $1.3458(0.0002)$ & $1.3598(0.0004)$ \\
\hline
\end{tabular}




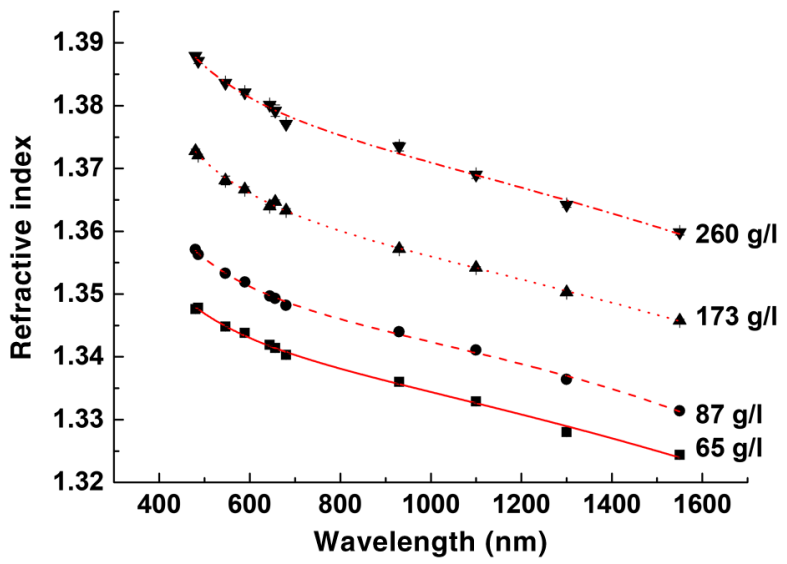

Fig. 3 The dispersion curves for hemoglobin solutions: the symbols are experimental data from Table 1 and the lines correspond to the fit of these data to the Sellmeier formula, Eq. (7).

Table 2 Coefficients of Sellmeier formula for hemoglobin solutions of different concentrations.

\begin{tabular}{lccccc}
$\mathrm{Hb}(\mathrm{g} / \mathrm{l})$ & $A 1$ & $A_{2}$ & $B 1\left(1 / \mathrm{nm}^{2}\right)$ & $B 2,10^{7}\left(1 / \mathrm{nm}^{2}\right)$ & $R^{2}$ \\
\hline 65 & 0.79099 & 685.08237 & 8366.45239 & 4024.35 & 0.995 \\
87 & 0.80835 & 450.24119 & 9983.69749 & 2842.83 & 0.999 \\
173 & 0.84507 & 402.89873 & 11065.32117 & 2540.72 & 0.998 \\
260 & 0.88871 & 190.95319 & 10187.17167 & 1039.98 & 0.993 \\
\hline
\end{tabular}

Table 3 presents data for the RI of distilled water and the specific increment of RI for hemoglobin solutions obtained by hemolysis. Approximation of the dependence of the specific increment of the RI on the wavelength was performed using the software package OriginProLab. The best fit was achieved using

$y=\frac{C x}{(D+x)}$

where $C=0.17263 \pm 0.00157$ and $D=-57.8324 \pm 5.56032$. The correlation coefficient was $R^{2}=0.90$.
Table 3 The distilled water RI $n_{\mathrm{H}_{2} \mathrm{O}}$ and the specific increment $\mathrm{d} n / \mathrm{d} C$ of $\mathrm{Rl}$ for hemoglobin solutions obtained by hemolysis, for the room temperature $23^{\circ} \mathrm{C}$. SD is shown in brackets.

\begin{tabular}{lccc}
$\lambda(\mathrm{nm})$ & $n_{\mathrm{H}_{2} \mathrm{O}}$ & $\alpha(\mathrm{ml} / \mathrm{g})$ & $\beta(\mathrm{ml} / \mathrm{g})$ \\
\hline 480 & $1.3371(0.0003)$ & $0.199(0.006)$ & $0.149(0.005)$ \\
486 & $1.3371(0.0002)$ & $0.196(0.005)$ & $0.147(0.004)$ \\
546 & $1.3342(0.0001)$ & $0.193(0.005)$ & $0.144(0.004)$ \\
589 & $1.3329(0.0002)$ & $0.192(0.005)$ & $0.144(0.003)$ \\
644 & $1.3313(0.0002)$ & $0.189(0.004)$ & $0.142(0.003)$ \\
656 & $1.3308(0.0002)$ & $0.190(0.005)$ & $0.143(0.003)$ \\
680 & $1.3301(0.0001)$ & $0.185(0.005)$ & $0.139(0.004)$ \\
930 & $1.3259(0.0002)$ & $0.183(0.004)$ & $0.138(0.003)$ \\
1100 & $1.3222(0.0002)$ & $0.183(0.005)$ & $0.139(0.004)$ \\
1300 & $1.3174(0.0002)$ & $0.185(0.006)$ & $0.140(0.004)$ \\
1550 & $1.3140(0.0002)$ & $0.179(0.004)$ & $0.136(0.003)$ \\
\hline
\end{tabular}

\section{Discussion}

The results of the measurements revealed that there is a linear relationship between the RI and hemoglobin concentration. Table 4 summarizes data on hemoglobin RI available in the literature. The comparison of received data with the literature is presented.

There is lack of data on the RI measurement of hemoglobin solutions for concentrations close to that in the RBC; specifically, data for the NIR region are practically absent. The RI of hemoglobin solution of $260 \mathrm{~g} / \mathrm{l}$, obtained from whole blood at room temperature $\left(23^{\circ} \mathrm{C}\right)$ for the wavelength of $480 \mathrm{~nm}$, was found to be equal to $1.3879 \pm 0.0002$, for $589 \mathrm{~nm}$ to $1.3821 \pm 0.0004$, for $1100 \mathrm{~nm}$ to $1.3690 \pm 0.0006$, and for $1550 \mathrm{~nm}$ to $1.3598 \pm 0.0002$. The concentration increment of RI of hemoglobin was found as $0.199 \pm 0.006 \mathrm{ml} / \mathrm{g}$ for the wavelength $480 \mathrm{~nm}, 0.192 \pm 0.005 \mathrm{ml} / \mathrm{g}$ for the wavelength

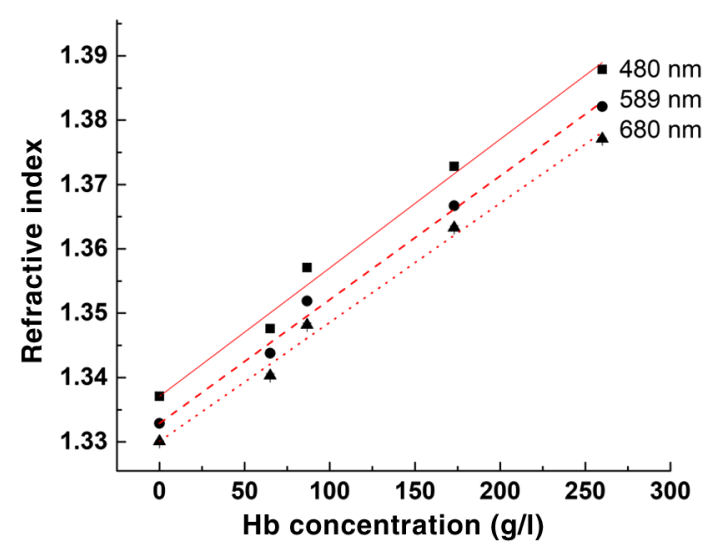

(a)

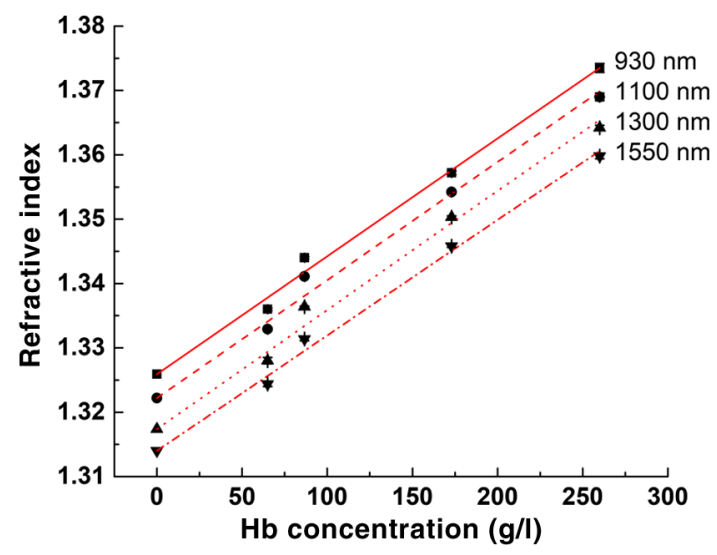

(b)

Fig. 4 The dependence of the RI on the concentration of hemoglobin in solution for: (a) visible and (b) NIR ranges (black symbols, experimental data; red lines, approximation of these data). 
Table 4 The experimental data for the real part of the RI of the hemoglobin solutions.

\begin{tabular}{|c|c|c|c|c|c|c|c|c|c|}
\hline & & & Notor & & $\lambda(\mathrm{nm})$ & $\mathrm{g} / \mathrm{l}$ & $N$ & Notes & Ref. \\
\hline$\lambda(\mathrm{nm})$ & $\mathrm{g} / \mathrm{l}$ & $N$ & Notes & Rer. & \multirow{3}{*}{450} & \multirow{3}{*}{320} & \multirow{3}{*}{1.3888} & \multirow{10}{*}{$\begin{array}{c}\text { Bovine hemoglobin } \\
\text { (lyophilized powder); } \\
0.5 \% \mathrm{HbO}_{2} ; T=20^{\circ} \mathrm{C} ; \\
\text { fiber spectrometer } \\
\text { Bovine hemoglobin } \\
\text { (lyophilized powder); } \\
\mathrm{Hb} ; T=20^{\circ} \mathrm{C} ; \\
\text { fiber spectrometer } \\
\text { Human hemoglobin from } \\
\text { whole blood; } \mathrm{HbO}_{2} ; \\
T=23^{\circ} \mathrm{C} ; \text { Multiwavelength } \\
\text { Abbe refractometer }\end{array}$} & \multirow[t]{6}{*}{22} \\
\hline \multirow[t]{4}{*}{250} & 46 & 1.398 & \multirow{12}{*}{$\begin{array}{l}\text { Human hemoglobin from } \\
\text { fresh RBC suspensions } \\
\text { of donors; VIS-NIR- } \\
\text { spectrometer }\end{array}$} & \multirow[t]{12}{*}{55,56} & & & & & \\
\hline & 104 & 1.406 & & & & & & & \\
\hline & 165 & 1.435 & & & \multirow[t]{3}{*}{450} & \multirow[t]{3}{*}{320} & \multirow[t]{2}{*}{1.3933} & & \\
\hline & 287 & 1.470 & & & & & & & \\
\hline \multirow[t]{4}{*}{300} & 46 & 1.373 & & & & & & & \\
\hline & 104 & 1.389 & & & \multirow[t]{4}{*}{480} & 65 & $1.3476(0.0003)$ & & a \\
\hline & 165 & 1.405 & & & & 87 & $1.3571(0.0003)$ & & \\
\hline & 287 & 1.441 & & & & 173 & $1.3728(0.0003)$ & & \\
\hline \multirow[t]{4}{*}{400} & 46 & 1.354 & & & & 260 & $1.3879(0.0002)$ & & \\
\hline & 104 & 1.367 & & & \multirow[t]{3}{*}{486} & 65 & $1.3478(0.0002)$ & \multirow{3}{*}{$\begin{array}{l}\text { Human hemoglobin from } \\
\text { whole blood; } \mathrm{HbO}_{2} ; \\
T=23^{\circ} \mathrm{C} ; \text { Multiwavelength } \\
\text { Abbe refractometer }\end{array}$} & a \\
\hline & 165 & 1.383 & & & & 87 & $1.3563(0.0002)$ & & \\
\hline & 287 & 1.409 & & & & 173 & $1.3721(0.0002)$ & & \\
\hline \multirow[t]{8}{*}{400} & 20 & 1.35223 & \multirow{8}{*}{$\begin{array}{l}\text { Bovine hemoglobin (dry); } \\
\text { Hb; } \mathrm{pH} \text { 7.4; room } \\
\text { temperature; continuous } \\
\text { RI dispersion (CRID) }\end{array}$} & \multirow[t]{16}{*}{47} & & 260 & $1.3871(0.0004)$ & & \\
\hline & 40 & 1.35495 & & & 486.1 & 140 & 1.361 & Human hemoglobin & 4,46 \\
\hline & 60 & 1.35806 & & & & & & $\begin{array}{l}\text { (lyophilized powder); Hb; } \\
T=20^{\circ} \mathrm{C} ; \mathrm{pH} 7.4 ; \mathrm{TIR}\end{array}$ & \\
\hline & 80 & 1.36078 & & & 486.1 & 140 & 1.361 & Human hemoglobin & \\
\hline & 120 & 1.36369 & & & & & & $\begin{array}{c}\text { (lyophilized powder); } \mathrm{HbO}_{2} \\
T=20^{\circ} \mathrm{C} ; \mathrm{pH} \text { 7.4; } \mathrm{TIR}\end{array}$ & \\
\hline & 140 & 1.36600 & & & 500 & 287 & 1.413 & Human hemoglobin from & 55,56 \\
\hline & 280 & 1.37010 & & & & 165 & 1.383 & fresh RBC suspensions of & \\
\hline & 320 & 1.38621 & & & & 104 & 1.363 & $\begin{array}{l}\text { donors; VIS-NIR- } \\
\text { spectrometer }\end{array}$ & \\
\hline \multirow[t]{8}{*}{400} & 20 & 1.35107 & \multirow{8}{*}{$\begin{array}{l}\text { Bovine hemoglobin (dry); } \\
\mathrm{HbO}_{2} ; \mathrm{pH} 7.4 ; \text { room } \\
\text { temperature; CRID }\end{array}$} & & & & 1348 & & \\
\hline & 40 & 1.35417 & & & & 46 & 1.348 & & \\
\hline & 60 & 1.35767 & & & 500 & 20 & 1.34583 & Bovine hemoglobin (dry); & 47 \\
\hline & 80 & 1.36039 & & & & 40 & 1.34913 & temperature; CRID & \\
\hline & 120 & 1.36369 & & & & 60 & 1.35223 & & \\
\hline & 140 & 1.36602 & & & & 80 & 1.35592 & & \\
\hline & 280 & 1.36951 & & & & 120 & 1.35922 & & \\
\hline & 320 & 1.38660 & & & & 140 & 1.36175 & & \\
\hline 400 & 320 & 1.3822 & Bovine hemoglobin & 26 & & 280 & 1.36544 & & \\
\hline & & & (lyophilized powder); & & & 320 & 1.38408 & & \\
\hline & & & $\begin{array}{l}0.5 \% \mathrm{HbO}_{2} ; T=20^{\circ} \mathrm{C} ; \\
\quad \text { fiber spectrometer }\end{array}$ & & 500 & 20 & 1.34505 & Bovine hemoglobin (dry); & \\
\hline 400 & 320 & 1.3775 & Bovine hemoglobin & & & 40 & 1.34854 & $\begin{array}{l}\mathrm{HbO}_{2} ; \mathrm{pH} \text { 7.4; room } \\
\text { temperature; CRID }\end{array}$ & \\
\hline & & & $\begin{array}{l}\text { (lyophilized powder); } \\
\text { Hb: } T=20^{\circ} \mathrm{C}\end{array}$ & & & 60 & 1.35262 & & \\
\hline & & & fber spectrometer & & & 80 & 1.35573 & & \\
\hline 401 & 140 & 1.365 & Human hemoglobin & 4,46 & & 120 & 1.35845 & & \\
\hline 435.8 & & 1.367 & $\begin{array}{c}\text { (lyophilized powder); Hb; } \\
T=20^{\circ} \mathrm{C} ; \mathrm{pH} 7.4 ; \mathrm{TIR}\end{array}$ & & & 140 & 1.36214 & & \\
\hline 401 & 140 & 1.369 & $\begin{array}{l}\text { (total internal reflection) } \\
\text { Human hemoglobin }\end{array}$ & & & 280 & 1.36544 & & \\
\hline 435.8 & & 1.366 & (lyophilized powder); $\mathrm{HbO}_{2}$ & & & 320 & 1.38505 & & \\
\hline 436 & & & $T=20^{\circ} \mathrm{C} ; \mathrm{pH} 7.4 ; \mathrm{TIR}$ & & 513.9 & 150 & 1.36053 & & 48 \\
\hline 436 & 150 & 1.36481 & $\begin{array}{l}\text { Human hemoglobin (dry); } \\
T=20^{\circ} \mathrm{C} ; \mathrm{pH} 7.4 ;\end{array}$ & 48 & & & & $\begin{array}{c}T=20^{\circ} \mathrm{C} ; \mathrm{pH} 7.4 \\
\text { Abbemat refractometer }\end{array}$ & 40 \\
\hline 438 & 140 & 1.374 & $\begin{array}{l}\text { AbDemat retractometer } \\
\text { Bovine hemoglobin (dry); }\end{array}$ & 47 & 532 & 1.7 & 1.3400 & Human hemoglobin & 37 \\
\hline & & & $\mathrm{Hb} ; \mathrm{HbO}_{2}$; room & & & 2.5 & 1.3431 & $\begin{array}{l}\text { (fresh human blood); } \\
T=25^{\circ} \mathrm{C} ; \mathrm{TIR}\end{array}$ & \\
\hline 440 & 50 & 1.3562 & $\begin{array}{l}\text { temperature; pH } 7.4 \\
\text { Human hemoglobin }\end{array}$ & 41 & & 4 & 1.3485 & & \\
\hline & 150 & 1.3780 & (lyophilized powder); & & & 7 & 1.3604 & & \\
\hline & 300 & 1.4187 & $\begin{array}{l}\text { spectroscopic phase } \\
\text { microscopy }\end{array}$ & & & 12.97 & 1.3871 & & \\
\hline
\end{tabular}

Table 4 (Continued). 


\begin{tabular}{|c|c|c|c|c|c|c|c|c|c|}
\hline$\lambda(\mathrm{nm})$ & $\mathrm{g} / \mathrm{l}$ & $N$ & Notes & Ref. & $\lambda(\mathrm{nm})$ & $g / l$ & $N$ & Notes & Ref. \\
\hline \multirow[t]{4}{*}{546} & 65 & $1.3448(0.0002)$ & \multirow{4}{*}{$\begin{array}{l}\text { Human hemoglobin from } \\
\text { whole blood; } \mathrm{HbO}_{2} ; \\
T=23^{\circ} \mathrm{C} \text {; Multiwavelength } \\
\text { Abbe refractometer }\end{array}$} & \multirow{4}{*}{ 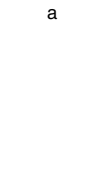 } & 600 & 20 & 1.34233 & \multirow{8}{*}{$\begin{array}{l}\text { Bovine hemoglobin (dry); } \\
\text { Hb; pH 7.4; room } \\
\text { temperature; CRID }\end{array}$} & \multirow[t]{15}{*}{47} \\
\hline & 87 & $1.3533(0.0002)$ & & & & 40 & 1.34485 & & \\
\hline & 173 & $1.3681(0.0007)$ & & & & 60 & 1.34874 & & \\
\hline & 260 & $1.3836(0.0002)$ & & & & 80 & 1.34835 & & \\
\hline \multirow[t]{3}{*}{546} & 50 & 1.3472 & \multirow{4}{*}{$\begin{array}{l}\text { Human hemoglobin } \\
\text { (lyophilized powder); } \\
\text { spectroscopic phase } \\
\text { microscopy }\end{array}$} & \multirow{3}{*}{41} & & 120 & 1.3520 & & \\
\hline & 150 & 1.3700 & & & & 140 & 1.35495 & & \\
\hline & 300 & 1.4051 & & & & 280 & 1.36155 & & \\
\hline \multirow{2}{*}{546.1} & \multirow{2}{*}{140} & \multirow{2}{*}{1.357} & & \multirow{4}{*}{4,46} & & 320 & 1.38233 & & \\
\hline & & & \multirow{3}{*}{$\begin{array}{c}\text { Human hemoglobin } \\
\text { (lyophilized powder); } \mathrm{Hb} ; \\
T=20^{\circ} \mathrm{C} ; \mathrm{pH} 7.4 ; \mathrm{TIR} \\
\text { Human hemoglobin } \\
\text { (lyophilized powder); } \mathrm{HbO}_{2} ; \\
T=20^{\circ} \mathrm{C} ; \mathrm{pH} 7.4 ; \mathrm{TIR}^{2}\end{array}$} & & 600 & $\begin{array}{l}20 \\
40\end{array}$ & $\begin{array}{l}1.34136 \\
1.34447\end{array}$ & \multirow{7}{*}{$\begin{array}{l}\text { Bovine hemoglobin (dry); } \\
\mathrm{HbO}_{2} ; \mathrm{pH} 7.4 ; \text { room } \\
\text { temperature; CRID }\end{array}$} & \\
\hline \multirow[t]{2}{*}{546.1} & \multirow[t]{2}{*}{140} & \multirow[t]{2}{*}{1.357} & & & & 60 & 1.34874 & & \\
\hline & & & & & & 80 & 1.35068 & & \\
\hline \multirow{3}{*}{550} & \multirow{3}{*}{320} & \multirow{3}{*}{1.3724} & \multirow{5}{*}{$\begin{array}{c}\text { Bovine hemoglobin } \\
\text { (lyophilized powder); } 0.5 \% \\
\mathrm{HbO}_{2} ; T=20^{\circ} \mathrm{C} \text {; fiber } \\
\text { spectrometer } \\
\text { Bovine hemoglobin } \\
\text { (lyophilized powder); } \mathrm{Hb} \text {; } \\
T=20^{\circ} \mathrm{C} ; \text { fiber } \\
\text { spectrometer }\end{array}$} & \multirow{5}{*}{26} & & 120 & 1.35456 & & \\
\hline & & & & & & 140 & 1.35767 & & \\
\hline & & & & & & 280 & 1.36155 & & \\
\hline \multirow[t]{2}{*}{550} & \multirow[t]{2}{*}{320} & 1.3738 & & & & 320 & 1.38058 & & \\
\hline & & & & & 600 & 320 & 1.3684 & $\begin{array}{l}\text { Bovine hemoglobin } \\
\text { (lyophilized powder); } \\
0.5 \% \mathrm{HbO}_{2} ; T=20^{\circ} \mathrm{C} \text {; }\end{array}$ & 26 \\
\hline 560 & 50 & 1.3466 & Human hemoglobin & 41 & 600 & 320 & 1.3702 & Bovine hemoglobin & \\
\hline & 150 & 1.3687 & $\begin{array}{l}\text { (lyophilized powder); } \\
\text { spectroscopic phase }\end{array}$ & & & & & (lyophilized powder); & \\
\hline & 300 & 1.4033 & microscopy & & & & & $\begin{array}{l}\mathrm{Hb} ; \mathrm{I}=20^{\circ} \mathrm{C} ; \text { tiber } \\
\text { spectrometer }\end{array}$ & \\
\hline 580 & $\begin{array}{c}50 \\
150\end{array}$ & $\begin{array}{l}1.3451 \\
13668\end{array}$ & $\begin{array}{l}\text { Human hemoglobin } \\
\text { (lyophilized powder); }\end{array}$ & 41 & 632 & 1.7 & 1.3626 & Human hemoglobin & 37 \\
\hline & 150 & 1.3668 & spectroscopic phase & & & 2.5 & 1.3360 & (fresh human blood); & \\
\hline & 300 & 1.4025 & microscopy & & & 4 & 1.3425 & $I=25 \mathrm{C} ; \mathrm{IR}$ & \\
\hline 587.6 & 140 & 1.356 & Human hemoglobin & 4,46 & & 7 & 1.3538 & & \\
\hline & & & $\begin{array}{l}\text { (lyophilized powder); Hb; } \\
T=20^{\circ} \mathrm{C} ; \mathrm{pH} 7.4 ; \mathrm{TIR}\end{array}$ & & & 12.97 & 1.3800 & & \\
\hline 587.6 & 140 & 1.357 & $\begin{array}{l}\text { Human hemoglobin } \\
\text { (lyophilized powder); } \mathrm{HbO}_{2} ; \\
T=20^{\circ} \mathrm{C} ; \mathrm{pH} 7.4 ; \mathrm{TIR}\end{array}$ & & $\begin{array}{l}632.8 \\
656.3\end{array}$ & 140 & $\begin{array}{l}1.354 \\
1.354\end{array}$ & $\begin{array}{l}\text { Human hemoglobin } \\
\text { (lyophilized powder); } \mathrm{Hb} \text {; } \\
T=20^{\circ} \mathrm{C} \text {; } \mathrm{pH} 7.4 ; \mathrm{TIR}\end{array}$ & 4,46 \\
\hline 589 & 65 & $1.3438(0.0002)$ & Human hemoglobin from & a & $\begin{array}{l}632.8 \\
656.3\end{array}$ & 140 & $\begin{array}{l}1.355 \\
1.354\end{array}$ & $\begin{array}{l}\text { Human hemoglobin } \\
\text { (lyophilized powder); } \mathrm{HbO}_{2}\end{array}$ & \\
\hline & 87 & $1.3519(0.0003)$ & $\begin{array}{c}\text { whole blood; } \mathrm{HbO}_{2} \\
T=23^{\circ} \mathrm{C}: \text { multiwavelength }\end{array}$ & & & & & $T=20^{\circ} \mathrm{C} ; \mathrm{pH} 7.4 ; \mathrm{TIR}$ & \\
\hline & 173 & $1.3667(0.0004)$ & Abbe refractometer & & $\begin{array}{l}633.2 \\
657.2\end{array}$ & 150 & $\begin{array}{l}1.35601 \\
1.35587\end{array}$ & $\begin{array}{l}\text { Human hemoglobin (dry); } \\
\qquad T=20^{\circ} \mathrm{C} ; \mathrm{pH} 7.4\end{array}$ & 48 \\
\hline & 260 & $1.3821(0.0004)$ & & & & & & Abbemat refractometer & \\
\hline 589 & 46 & 1.343 & Human hemoglobin from & 55,56 & 633 & $\begin{array}{l}104 \\
165\end{array}$ & $\begin{array}{l}1.3600 \\
1.3750\end{array}$ & $\begin{array}{l}\text { Human hemoglobin (dry); } \\
\qquad T=20^{\circ} \mathrm{C} ; \mathrm{pH} 7.4 ;\end{array}$ & 56 \\
\hline & 104 & 1.357 & $\begin{array}{c}\text { fresh RBC suspensions of } \\
\text { donors; VIS-NIR- }\end{array}$ & & 644 & & & Abbemat refractometer & a \\
\hline & 165 & 1.375 & spectrometer & & 044 & 87 & $1.3497(0.0002)$ & whole blood; $\mathrm{HbO}_{2}$ & \\
\hline & 287 & 1.406 & & & & 173 & $1.3640(0.0003)$ & $\begin{array}{c}T=23^{\circ} \mathrm{C} ; \text { multiwavelength } \\
\text { Abbe refractometer }\end{array}$ & \\
\hline 589.2 & 150 & 1.35724 & Human hemoglobin (dry); & 48 & & 260 & $1.3801(0.0003)$ & & \\
\hline & & & $\begin{array}{l}T=20^{\circ} \mathrm{C} ; \mathrm{pH} 7.4 ; \\
\text { Abbemat refractometer }\end{array}$ & & 650 & 320 & 1.3652 & $\begin{array}{l}\text { Bovine hemoglobin } \\
\text { (lyophilized powder): }\end{array}$ & 26 \\
\hline 589.3 & 140 & 1.356 & $\begin{array}{l}\text { Human hemoglobin } \\
\text { (lyophilized powder); Hb; } \\
T=20^{\circ} \mathrm{C} ; \mathrm{pH} 7.4 ; \mathrm{TIR}\end{array}$ & 4,46 & & & & $\begin{array}{l}0.5 \% \mathrm{HbO}_{2} ; T=20^{\circ} \mathrm{C} ; \\
\quad \text { fiber spectrometer }\end{array}$ & \\
\hline 589.3 & 140 & 1.357 & $\begin{array}{l}\text { Human hemoglobin } \\
\text { (lyophilized powder); } \mathrm{HbO}_{2} ; \\
T=20^{\circ} \mathrm{C} ; \mathrm{pH} 7.4 ; \mathrm{TIR}\end{array}$ & & 650 & 320 & 1.3668 & $\begin{array}{l}\text { Bovine hemoglobin } \\
\text { (lyophilized powder); } \\
\mathrm{Hb} ; T=20^{\circ} \mathrm{C} \text {; fiber } \\
\text { spectrometer }\end{array}$ & \\
\hline 600 & 50 & 1.3443 & Human hemoglobin & 41 & 655 & 50 & 1.3408 & Human hemoglobin & 41 \\
\hline & 150 & 1.3666 & $\begin{array}{l}\text { (lyophilized powder); } \\
\text { spectroscopic phase }\end{array}$ & & & 150 & 1.3642 & (lyophilized powder); & \\
\hline & 300 & 1.4014 & microscopy & & & 300 & 1.3969 & $\begin{array}{l}\text { spectroscopic phase } \\
\text { microscopy }\end{array}$ & \\
\hline
\end{tabular}


Table 4 (Continued).

\begin{tabular}{|c|c|c|c|c|}
\hline$\lambda(\mathrm{nm})$ & $g / l$ & $N$ & Notes & Ref. \\
\hline \multirow[t]{4}{*}{656} & 65 & $1.3414(0.0002)$ & \multirow{4}{*}{$\begin{array}{l}\text { Human hemoglobin from } \\
\text { whole blood; } \mathrm{HbO}_{2} \\
T=23^{\circ} \mathrm{C} ; \text { multiwavelength } \\
\text { Abbe refractometer }\end{array}$} & \multirow[t]{4}{*}{$a$} \\
\hline & 87 & $1.3493(0.0002)$ & & \\
\hline & 173 & $1.3647(0.0003)$ & & \\
\hline & 260 & $1.3792(0.0009)$ & & \\
\hline \multirow[t]{4}{*}{680} & 65 & $1.3403(0.0003)$ & \multirow{4}{*}{$\begin{array}{l}\text { Human hemoglobin from } \\
\text { whole blood; } \mathrm{HbO}_{2} ; \\
T=23^{\circ} \mathrm{C} ; \text { multiwavelength } \\
\text { Abbe refractometer }\end{array}$} & \multirow{4}{*}{ 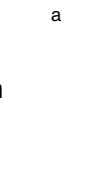 } \\
\hline & 87 & $1.3482(0.0003)$ & & \\
\hline & 173 & $1.3633(0.0003)$ & & \\
\hline & 260 & $1.3771(0.0002)$ & & \\
\hline \multirow[t]{3}{*}{700} & 50 & 1.3405 & \multirow{3}{*}{$\begin{array}{l}\text { Human hemoglobin } \\
\text { (lyophilized powder); } \\
\text { spectroscopic phase } \\
\text { microscopy }\end{array}$} & \multirow[t]{3}{*}{41} \\
\hline & 150 & 1.3634 & & \\
\hline & 300 & 1.3971 & & \\
\hline \multirow[t]{8}{*}{700} & 20 & 1.33961 & \multirow{8}{*}{$\begin{array}{c}\text { Bovine hemoglobin (dry); } \\
\mathrm{Hb} \text {; pH 7.4; room } \\
\text { temperature; CRID }\end{array}$} & \multirow[t]{8}{*}{47} \\
\hline & 40 & 1.34252 & & \\
\hline & 60 & 1.34602 & & \\
\hline & 80 & 1.34874 & & \\
\hline & 120 & 1.35184 & & \\
\hline & 140 & 1.35456 & & \\
\hline & 280 & 1.35806 & & \\
\hline & 320 & 1.37709 & & \\
\hline \multirow[t]{8}{*}{700} & 20 & 1.33883 & \multirow{8}{*}{$\begin{array}{l}\text { Bovine hemoglobin (dry); } \\
\mathrm{HbO}_{2} ; \mathrm{pH} 7.4 \text {; room } \\
\text { temperature; CRID }\end{array}$} & \\
\hline & 40 & 1.34175 & & \\
\hline & 60 & 1.34583 & & \\
\hline & 80 & 1.34835 & & \\
\hline & 120 & 1.35107 & & \\
\hline & 140 & 1.35476 & & \\
\hline & 280 & 1.35748 & & \\
\hline & 320 & 1.3767 & & \\
\hline 700 & 320 & 1.3612 & $\begin{array}{l}\text { Bovine hemoglobin } \\
\text { (lyophilized powder); } \\
0.5 \% \mathrm{HbO}_{2} ; T=20^{\circ} \mathrm{C} \text {; } \\
\text { fiber spectrometer }\end{array}$ & 26 \\
\hline 700 & 320 & 1.3637 & $\begin{array}{l}\text { Bovine hemoglobin } \\
\text { (lyophilized powder); } \\
\text { Hb; } T=20^{\circ} \mathrm{C} \text {; fiber } \\
\text { spectrometer }\end{array}$ & \\
\hline \multirow[t]{4}{*}{700} & 46 & 1.341 & \multirow{4}{*}{$\begin{array}{l}\text { Human hemoglobin from } \\
\text { fresh RBC suspensions of } \\
\text { donors; VIS-NIR- } \\
\text { spectrometer }\end{array}$} & \multirow[t]{4}{*}{55,56} \\
\hline & 104 & 1.356 & & \\
\hline & 165 & 1.374 & & \\
\hline & 287 & 1.404 & & \\
\hline 706.5 & 140 & 1.352 & \multirow{2}{*}{$\begin{array}{c}\text { Human hemoglobin } \\
\text { (lyophilized powder); } \mathrm{Hb} ; \\
T=20^{\circ} \mathrm{C} ; \mathrm{pH} 7.4 ; \mathrm{TIR} \\
\text { Human hemoglobin } \\
\text { (lyophilized powder); } \mathrm{HbO}_{2} ; \\
T=20^{\circ} \mathrm{C} ; \mathrm{pH} 7.4 ; \mathrm{TIR}\end{array}$} & \multirow[t]{2}{*}{4,46} \\
\hline 706.5 & 140 & 1.352 & & \\
\hline 750 & 320 & 1.3589 & $\begin{array}{l}\text { Bovine hemoglobin } \\
\text { (lyophilized powder); } \\
0.5 \% \mathrm{HbO}_{2} ; T=20^{\circ} \mathrm{C} ; \\
\text { fiber spectrometer }\end{array}$ & 26 \\
\hline 750 & 320 & 1.3599 & $\begin{array}{l}\text { Bovine hemoglobin } \\
\text { (lyophilized powder); } \\
\mathrm{Hb} ; T=20^{\circ} \mathrm{C} ; \text { Fiber } \\
\text { spectrometer }\end{array}$ & \\
\hline
\end{tabular}

Table 4 (Continued).

\begin{tabular}{|c|c|c|c|c|}
\hline$\lambda(\mathrm{nm})$ & $g / l$ & $N$ & Notes & Ref. \\
\hline \multirow[t]{4}{*}{800} & 46 & 1.338 & \multirow{4}{*}{$\begin{array}{l}\text { Human hemoglobin from } \\
\text { fresh RBC suspensions of } \\
\text { donors; VIS-NIR- } \\
\text { spectrometer }\end{array}$} & \multirow{4}{*}{55,56} \\
\hline & 104 & 1.353 & & \\
\hline & 165 & 1.370 & & \\
\hline & 287 & 1.400 & & \\
\hline \multirow[t]{4}{*}{900} & 46 & 1.338 & \multirow{4}{*}{$\begin{array}{l}\text { Human hemoglobin from } \\
\text { fresh RBC suspensions of } \\
\text { donors; VIS-NIR- } \\
\text { spectrometer }\end{array}$} & \multirow{4}{*}{55,56} \\
\hline & 104 & 1.352 & & \\
\hline & 165 & 1.369 & & \\
\hline & 287 & 1.401 & & \\
\hline \multirow[t]{4}{*}{930} & 65 & $1.3360(0.0002)$ & \multirow{4}{*}{$\begin{array}{l}\text { Human hemoglobin from } \\
\text { whole blood; } \mathrm{HbO}_{2} ; \\
T=23^{\circ} \mathrm{C} ; \text { Multiwavelength } \\
\text { Abbe refractometer }\end{array}$} & \multirow[t]{4}{*}{ a } \\
\hline & 87 & $1.3440(0.0002)$ & & \\
\hline & 173 & $1.3572(0.0003)$ & & \\
\hline & 260 & $1.3735(0.0007)$ & & \\
\hline \multirow[t]{4}{*}{1000} & 46 & 1.338 & \multirow{4}{*}{$\begin{array}{l}\text { Human hemoglobin from } \\
\text { fresh RBC suspensions of } \\
\text { donors; VIS-NIR- } \\
\text { spectrometer }\end{array}$} & \multirow{4}{*}{55,56} \\
\hline & 104 & 1.353 & & \\
\hline & 165 & 1.370 & & \\
\hline & 287 & 1.401 & & \\
\hline \multirow[t]{4}{*}{1100} & 46 & 1.337 & \multirow{4}{*}{$\begin{array}{l}\text { Human hemoglobin from } \\
\text { fresh RBC suspensions of } \\
\text { donors; VIS-NIR- } \\
\text { spectrometer }\end{array}$} & \multirow[t]{4}{*}{55,56} \\
\hline & 104 & 1.352 & & \\
\hline & 165 & 1.369 & & \\
\hline & 287 & 1.400 & & \\
\hline \multirow[t]{4}{*}{1100} & 65 & $1.3329(0.0002)$ & \multirow{4}{*}{$\begin{array}{l}\text { Human hemoglobin from } \\
\text { whole blood; } \mathrm{HbO}_{2} ; \\
T=23^{\circ} \mathrm{C} ; \text { Multiwavelength } \\
\text { Abbe refractometer }\end{array}$} & \multirow[t]{4}{*}{$\mathrm{a}$} \\
\hline & 87 & $1.3411(0.0002)$ & & \\
\hline & 173 & $1.3542(0.0002)$ & & \\
\hline & 260 & $1.3690(0.0006)$ & & \\
\hline \multirow[t]{4}{*}{1300} & 65 & $1.3280(0.0005)$ & \multirow{4}{*}{$\begin{array}{l}\text { Human hemoglobin from } \\
\text { whole blood; } \mathrm{HbO}_{2} ; \\
T=23^{\circ} \mathrm{C} ; \text { Multiwavelength } \\
\text { Abbe refractometer }\end{array}$} & \multirow[t]{4}{*}{ a } \\
\hline & 87 & $1.3364(0.0002)$ & & \\
\hline & 173 & $1.3503(0.0002)$ & & \\
\hline & 260 & $1.3642(0.0004)$ & & \\
\hline \multirow[t]{4}{*}{1550} & 65 & $1.3244(0.0004)$ & \multirow{4}{*}{$\begin{array}{l}\text { Human hemoglobin from } \\
\text { whole blood; } \mathrm{HbO}_{2} ; \\
T=23^{\circ} \mathrm{C} ; \text { ultiwavelength } \\
\text { Abbe refractometer }\end{array}$} & \multirow[t]{4}{*}{$\mathrm{a}$} \\
\hline & 87 & $1.3314(0.0003)$ & & \\
\hline & 173 & $1.3458(0.0002)$ & & \\
\hline & 260 & $1.3598(0.0004)$ & & \\
\hline
\end{tabular}

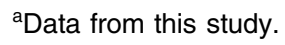

$589 \mathrm{~nm}, 0.183 \pm 0.005 \mathrm{ml} / \mathrm{g}$ for the wavelength $930 \mathrm{~nm}$, and $0.179 \pm 0.004 \mathrm{ml} / \mathrm{g}$ for the wavelength $1550 \mathrm{~nm}$.

Freibel et al. also measured the RI of a hemoglobin solution of $287 \mathrm{~g} / 1$ obtained from whole blood. According to their measurements using the spectral method and the Fresnel formula, the RI was 1.409 for the wavelength $400 \mathrm{~nm}, 1.406$ for the wavelength $589 \mathrm{~nm}, 1.404$ for the wavelength $700 \mathrm{~nm}$, and 1.400 for the wavelength $1100 \mathrm{~nm}^{55,56}$ The same scientific group received at the wavelength $633 \mathrm{~nm}$ the $\mathrm{RI}=1.3750$ for concentration $165 \mathrm{~g} / 1$ and the $\mathrm{RI}=1.3600$ for concentration 104 g/l. Jin et al., ${ }^{37}$ Park et al., ${ }^{41}$ Zhernovaya et al., ${ }^{46}$ Yahya et al., ${ }^{48}$ and Deng et al. ${ }^{47}$ used a solution obtained from dry hemoglobin for the study of refraction. Zhernovaya et al. measured the RI of oxygenated and deoxygenated hemoglobin of $140 \mathrm{~g} / 1$ by refractometer Abbe for nine wavelengths at a temperature of $20^{\circ} \mathrm{C}$. For example, the values of RI were 1.361 for the wavelength $486 \mathrm{~nm}, 1.357$ for the wavelength $589 \mathrm{~nm}$, and 


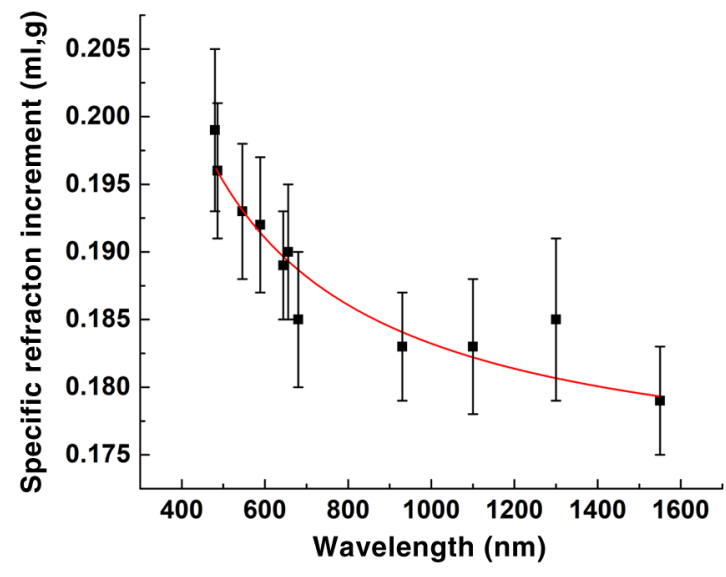

Fig. 5 The dependence of the specific RI increment $\alpha$ of hemoglobin solution on the wavelength (black symbols, estimated data, red lines, approximation of data).

1.352 for the wavelength $706.5 \mathrm{~nm} .{ }^{46}$ Yahya et al. measured RI of oxygenated human hemoglobin $150 \mathrm{~g} / 1$ as 1.36481 for the wavelength $436 \mathrm{~nm}, 1.35724$ for the wavelength $589 \mathrm{~nm}$, and 1.35587 for the wavelength $657.2 \mathrm{~nm} .{ }^{48}$ Deng et al. measured RI of $50 \%$ oxyhemoglobin $320 \mathrm{~g} / 1$ by fiber spectrometer at a temperature of $20^{\circ} \mathrm{C}$. RIs were 1.3775 for the wavelength $500 \mathrm{~nm}, 1.3684$ for the wavelength $600 \mathrm{~nm}$, and 1.3612 for the wavelength $700 \mathrm{~nm} .{ }^{47} \mathrm{Jin}$ et al. determined RI of hemoglobin for concentration of $12.97 \mathrm{mmol} / 1$ as 1.3871 for the wavelength $532 \mathrm{~nm}$ and 1.3800 for the wavelength $632 \mathrm{~nm} .{ }^{37}$ Park et al. measured the dispersion of $\mathrm{Hb}$ solutions, prepared from $\mathrm{Hb}$ protein powder, at three different concentrations: $0.05,0.15$, and $0.30 \mathrm{~g} / \mathrm{ml}$. For example, the RI for $0.15 \mathrm{~g} / \mathrm{ml}$ was 1.3687 at wavelength $560 \mathrm{~nm}$.

Zhernovaya et al., ${ }^{46}$ Freibel et al., ${ }^{55,56}$ Yahya et al., ${ }^{48}$ and Park et al. ${ }^{41}$ also calculated the specific increment of RI $\left(20^{\circ} \mathrm{C}\right)$, which was equal to $0.147,0.2015$, and $0.151 \mathrm{ml} / \mathrm{g}$ for the wavelength $589 \mathrm{~nm}$ and $0.183 \pm 0.003 \mathrm{ml} / \mathrm{g}$ for the wavelength range of 440 to $700 \mathrm{~nm}$, respectively. In this study, the RI-specific increment of hemoglobin was found as $0.192 \pm 0.005 \mathrm{ml} / \mathrm{g}$ for the wavelength $589 \mathrm{~nm}$ and temperature at $23^{\circ} \mathrm{C}$.

The discrepancy between literature and our data may be caused by the differences in the sample preparation protocols since the human hemoglobin may differ in content of various forms of hemoglobin of donor's blood. The specificities of experimental setups also may play a role.

In Fig. 5, it is seen that the RI-specific increment of a solution of human hemoglobin decreases with the wavelength. This could be explained by the dispersion theory of multicomponent materials and caused by strong absorption bands of hemoglobin and water in UV, hemoglobin in the visible, and water in the NIR. The dependence of the specific RI increment $\alpha$ of hemoglobin solution on the wavelength is in a good agreement with the literature data given by Friebel et al. for whole blood using an integrating sphere spectrometer technique and by Jung et al. for an $\mathrm{Hb}$ solution in intact individual RBC cytoplasm. ${ }^{43,55}$

As the experimental data for the real part of RI of hemoglobin solutions differ for measurements done by alternative techniques (see Table 4), it is important for researchers to use a specific tool, such as the Kramers-Kronig relations, to analyze experimental results for discrete wavelengths and to derive the RI real part theoretically from the measurements of its imaginary part. ${ }^{4,24,49,50,53}$ In addition to providing quantification of the real part of the RI of hemoglobin at selected wavelengths, where no direct measurements are available, they are independent of hemoglobin concentration and thus can augment the model functions for the RI found by alternative methods. ${ }^{4}$ Such analysis was done early in Ref. 4 for the measurements of the real part of the RI of hemoglobin solutions at eight discrete wavelengths from 400 to $700 \mathrm{~nm}$, and we received encouraging results. In this work, measurements were done in a wider wavelength range from 480 to $1550 \mathrm{~nm}$ at 11 discrete wavelengths, which will allow us to make a more precise Kramers-Kronig analysis, results of which we are planning to publish in the near future.

\section{Conclusion}

The RI of hemoglobin solutions has been measured for visible and NIR ranges using a commercially available multiwavelength Atago refractometer. Data were approximated by the Sellmeier formula with a high accuracy in a whole wavelength range. The absolute value of the initial index of refraction $n_{0}$ and the specific refraction increment $\mathrm{d} n / \mathrm{d} C$ on hemoglobin concentration $C$ for room temperature at $23^{\circ} \mathrm{C}$ were derived from these measurements for each wavelength from 480 to $1550 \mathrm{~nm}$. The data obtained are in good agreement with available data in the literature and supplementary to already measured values as done for new wavelengths, which allowed for evaluation of the specific refraction increment $\mathrm{d} n / \mathrm{d} C$ in a wide spectral range.

\section{Disclosures}

The authors have no relevant financial interests in this article and no potential conflicts of interest to disclose.

\section{Acknowledgments}

The authors appreciate support from the Tomsk State University Competitiveness Improvement Programme, from the 5 top 100 Russian Academic Excellence Project at the Immanuel Kant Baltic Federal University (ENL), grants of the RFBR 17-0200358 and the MES RF 17.1223.2017/AP (VVT).

\section{References}

1. J. J. J. Dirckx, L. C. Kuypers, and W. F. Decraemer, "Refractive index of tissue measured with confocalmicroscopy," J. Biomed. Opt. 10(4), 044014 (2005).

2. T. L. Troy, D. L. Page, and E. M. Sevick-Muraca, "Optical properties of normal and diseased breast tissues: prognosis for optical mammography," J. Biomed. Opt. 1(3), 342-355 (1996).

3. A. N. Bashkatov, E. A. Genina, and V. V. Tuchin, "Optical properties of skin, subcutaneous, and muscle tissues: a review," J. Innovative Opt. Health Sci. 4(1), 9-38 (2011)

4. O. Sydoruk et al., "Refractive index of solutions of human hemoglobin from the near-infrared to the ultraviolet range: Kramers-Kronig analysis," J. Biomed. Opt. 17(11), 115002 (2012).

5. L. V. Wang and H. I. Hu, Biomedical Optics: Principles and Imaging, John Wiley and Sons, Hoboken, New Jersey (2007).

6. V. V. Tuchin, Tissue Optics: Light Scattering Methods and Instruments for Medical Diagnostics, 3rd ed., PM 254, p. 988, SPIE Press, Bellingham, Washington (2015).

7. C. Meinke, M. Friebel, and J. Helfmann, "Optical properties of flowing blood cells," in Advanced Optical Flow Cytometry: Methods and Disease Diagnoses, V. V. Tuchin, Ed., pp. 95-132, Wiley-VCH Verlag GmbH \& Co. KGaA, Weinheim (2011).

8. M. Meinke et al., "Optical properties of platelets and blood plasma and their influence on the optical behavior of whole blood in the visible to near infrared wavelength range," J. Biomed. Opt. 12, 014024 (2007).

9. O. S. Zhernovaya, V. V. Tuchin, and I. V. Meglinski, "Monitoring of blood proteins glycation by refractive index and spectral measurements," Laser Phys. Lett. 5(6), 460-464 (2008). 
10. P. Giannios et al., "Complex refractive index of normal and malignant human colorectal tissue in the visible and near-infrared," J. Biophotonics 10(2), 303-310 (2017).

11. S. Carvalho et al., "Wavelength dependence of the refractive index of human colorectal tissues: comparison between healthy mucosa and cancer," J. Biomed. Photonics Eng. 2(4), 040307 (2016).

12. G. Popescu, Quantitative Phase Imaging of Cells and Tissues, McGrawHill, New York (2011).

13. H. Majeed et al., "Quantitative phase imaging for medical diagnosis," J. Biophotonics 10(2), 177-205 (2017).

14. M. Shan, M. E. Kandel, and G. Popescu, "Refractive index variance of cells and tissues measured by quantitative phase imaging," Opt. Express 25 (2), 1573-1581 (2017).

15. M. E. Kandel et al., "Label-free tissue scanner for colorectal cancer screening," J. Biomed. Opt. 22(6), 066016 (2017).

16. Z. Wang et al., "Tissue refractive index as marker of disease," J. Biomed. Opt. 16(11), 116017 (2011).

17. T. H. Nguyen et al., "Automatic Gleason grading of prostate cancer using quantitative phase imaging and machine learning," J. Biomed. Opt. 22, 036015 (2017).

18. H. Majeed et al., "Quantifying collagen fiber orientation in breast cancer using quantitative phase imaging," J. Biomed. Opt. 22, 046004 (2017).

19. M. Lee et al., "Label-free optical quantification of structural alterations in Alzheimer's disease," Sci. Rep. 6, 31034 (2016).

20. A. Greenbaum et al., "Wide-field computational imaging of pathology slides using lens-free on-chip microscopy," Sci. Transl. Med. 6, 267ra175 (2014).

21. M. V. Volkenshtein, Molecular Optics, p. 96, Gosteskhizdat, Moscow (1951).

22. G. M. Hale and M. R. Querry, "Optical constants of water in the 200-nm to 200- $\mu$ m wavelength region," Appl. Opt. 12(3), 555-563 (1973).

23. D. Segelstein, "The complex refractive index of water," MS Thesis, Department of Physics, University of Missouri, Kansas City (1981).

24. L. X. Cundin and W. P. Roach, "Kramers-Kronig analysis of biological skin," arXiv:1010.3752v1 (2010).

25. K. Lee et al., "Measurements of complex refractive indices of photoactive yellow protein," arXiv:1507.00412 (2015).

26. Z. Deng et al., "Determination of continuous complex refractive index dispersion of biotissue based on internal reflection," J. Biomed. Opt. 21(1), 015003 (2016).

27. F. P. Bolin et al., "Refractive index of some mammalian tissues using a fiber optic cladding method," Appl. Opt. 28(12), 2297-2303 (1989).

28. M. Daimon and A. Masumura, "Measurement of the refractive index of distilled water from the near-infrared region to the ultraviolet region," Appl. Opt. 46(18), 3811-3820 (2007).

29. D. K. Sardar and L. B. Levy, "Optical properties of whole blood," Lasers Med. Sci. 13(2), 106-111 (1998).

30. G. J. Tearney et al., "Determination of the refractive index of highly scattering human tissue by optical coherence tomography," Opt. Lett. 20, 2258-2260 (1995).

31. A. Knüttel, S. Bonev, and W. Knaak, "New method for evaluation of in vivo scattering and refractive index properties obtained with optical coherence tomography," J. Biomed. Opt. 9(2), 265-273 (2004).

32. H.-C. Cheng and Y.-C. Liu, "Simultaneous measurement of group refractive index and thickness of optical samples using optical coherence tomography," Appl. Opt. 49, 790-797 (2010).

33. I. Y. Yanina, N. A. Trunina, and V. V. Tuchin, "Photoinduced cell morphology alterations quantified within adipose tissues by spectral optical coherence tomography," J. Biomed. Opt. 18(11), 111407 (2013).

34. F. E. Robles, S. Chowdhury, and A. Wax, "Assessing hemoglobin concentration using spectroscopic optical coherence tomography for feasibility of tissue diagnostics," Biomed. Opt. Express 1(1), 310-317 (2010).

35. J. H. Jung et al., "Hyperspectral optical diffraction tomography," Opt. Express 24(3), 2006-2012 (2016).

36. F. E. Robles et al., "Molecular imaging true-colour spectroscopic optical coherence tomography," Nat. Photonics 5(12), 744-747 (2011).

37. Y. L. Jin et al., "Refractive index measurement for biomaterial samples by total internal reflection," Phys. Med. Biol. 51(20), N371-N379 (2006).
38. A. García-Valenzuela and H. Contreras-Tello, "Optical model enabling the use of Abbe-type refractometers on turbid suspensions," Opt. Lett. 38(5), 775-777 (2013).

39. H. Contreras-Tello and A. García-Valenzuela, "Refractive index measurement of turbid media by transmission of backscattered light near the critical angle," Appl. Opt. 53(21), 4768-4778 (2014).

40. N. Lue and G. Popescu, "Live cell refractometry using microfluidic devices," Opt. Lett. 31(18), 2759-2761 (2006).

41. Y. K. Park et al., "Spectroscopic phase microscopy for quantifying hemoglobin concentrations in intact red blood cells," Opt. Lett. 34(23), 3668-3670 (2009).

42. F. E. Robles, L. L. Satterwhite, and A. Wax, "Non-linear phase dispersion spectroscopy," Opt. Lett. 36(23), 4665-4667 (2011).

43. J.-H. Jung, J. Jang, and Y. K. Park, "Spectro-refractometry of individual microscopic objects using swept-source quantitative phase imaging," Anal. Chem. 85(21), 10519-10525 (2013).

44. R. Barer, "Refractometry and interferometry of living cells," J. Opt. Soc. Am. 47(6), 545-556 (1957)

45. D. J. Faber et al., "Oxygen saturation-dependent absorption and scattering of blood," Phys. Rev. Lett. 93(2), 028102 (2004).

46. O. Zhernovaya et al., "The refractive index of human hemoglobin in the visible range," Phys. Med. Biol. 56(13), 4013-4021 (2011).

47. J. Wang et al., "Measurement of the refractive index of hemoglobin solutions for a continuous spectral region," Biomed. Opt. Express 6(7), 2536-2541 (2015).

48. M. Yahya and M. Z. Saghir, "Empirical modelling to predict the refractive index of human blood," Phys. Med. Biol. 61, 1405-1415 (2016).

49. S. F. Shumilina, "Dispersion of real and imaginary part of the complex refractive index of hemoglobin in the range 450 to $820 \mathrm{~nm}$," Bullet. Beloruss. SSR Acad. Sci. 1, 79-84 (1984).

50. J. Gienger et al., "Determining the refractive index of human hemoglobin solutions by Kramers-Kronig relations with an improved absorption model," Appl. Opt. 55(31), 8951-8961 (2016).

51. G. V. Maksimov et al., "Role of viscosity and permeability of the erythrocyte plasma membrane in changes in oxygen-binding properties of hemoglobin during diabetes mellitus," Bull. Exp. Biol. Med. 140(5), 510-513 (2005).

52. J. Singh, Optical Properties of Condensed Matter and Applications, Wiley, Chichester (2006).

53. S. A. Prahl, "Optical absorption of hemoglobin," Oregon Medical Laser Center, http://omlc.ogi.edu/spectra/hemoglobin/index.html (3 March 2018).

54. M. Andersen and L. Painter, "Dispersion equation and polarizability of bovine serum albumin from measurements of refractive indices," Biopolymers 13, 1261-1267 (1974).

55. M. Friebel and M. Meinke, "Model function to calculate the refractive index of native hemoglobin in the wavelength range of 250 to $1100 \mathrm{~nm}$ dependent on concentration," Appl. Opt. 45(12), 2838-2842 (2006).

56. M. Friebel, "Determination of the complex refractive index of highly concentrated hemoglobin solutions using transmittance and reflectance measurements," J. Biomed. Opt. 10(6), 064019 (2005).

Ekaterina N. Lazareva is an engineer at the Department of Optics and Biophotonics of Saratov State University. Her research interests are in optical properties of biological tissues, especially of blood (its components) and adipose tissue, refractometry and spectroscopy of tissues, and tissue optical clearing.

Valery V. Tuchin is a professor and head of optics and biophotonics at Saratov State University (National Research University of Russia) and several other universities. His research interests include tissue optics, laser medicine, tissue optical clearing, and nanobiophotonics. He is a fellow of SPIE and OSA, has been awarded Honored Science Worker of the Russia, Honored Professor of Saratov University, SPIE Educator Award, FiDiPro, Finland, Chime Bell Prize of Hubei Province, China, NanQiang Life Science Series Lectures Award of Xiamen University, China, and the Joseph W. Goodman Book Writing Award (OSA/SPIE). 